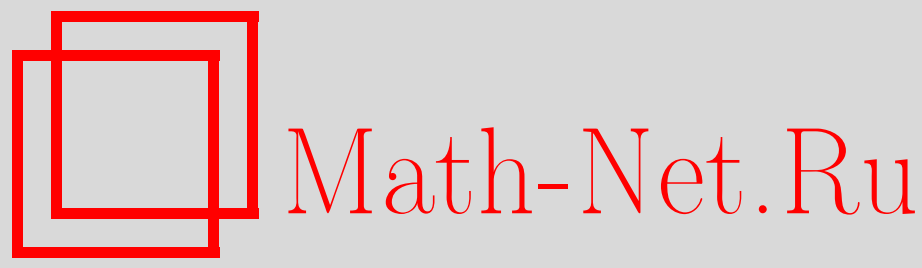

В. П. Михайлов, Достаточное условие существования предельных значений на границе у решений эллиптического уравнения, ТМФ, 2008, том 157, номер 3, 436-449

DOI: https://doi.org/10.4213/tmf6291

Использование Общероссийского математического портала Math-Net.Ru подразумевает, что вы прочитали и согласны с пользовательским соглашением http://www . mathnet.ru/rus/agreement

Параметры загрузки:

IP : 34.229 .108 .108

26 апреля 2023 г., 17:40:19

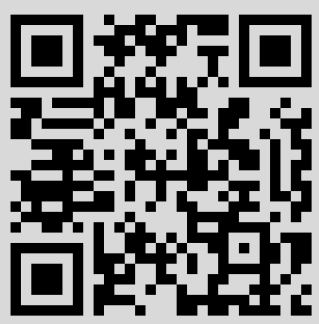




\section{ДОСТАТОЧНОЕ УСЛОВИЕ СУЩЕСТВОВАНИЯ ПРЕДЕЛЬНЫХ ЗНАЧЕНИЙ НА ГРАНИЦЕ У РЕШЕНИЙ ЭЛЛИПТИЧЕСКОГО УРАВНЕНИЯ}

Установлено достаточное условие существования предельного значения на границе области у решения эллиптического уравнения.

Ключевые слова: эллиптические уравнения, граница области, предельное значение на границе.

Настоящая работа примыкает к работе автора [1].

В полосе $\Pi=\left\{x=\left(x_{1}, \ldots, x_{n}\right) \in \mathbb{R}^{n}, 0<y<1\right\}$ пространства $\mathbb{R}^{n+1}$ рассматривается уравнение

$$
\mathfrak{P}(\Delta) u(x, y)=0, \quad(x, y) \in \Pi,
$$

где $\Delta=\frac{\partial^{2}}{\partial x_{1}^{2}}+\cdots+\frac{\partial^{2}}{\partial x_{n}^{2}}+\frac{\partial^{2}}{\partial y^{2}}-$ оператор Лапласа, a $\mathfrak{P}(z)-$ многочлен степени $m \geqslant 1$ с постоянными комплексными коэффициентами. Пусть $z_{1}, \ldots, z_{k}$ - попарно различные корни этого многочлена, $z_{i} \neq z_{j}$ при $i \neq j$, а $m_{1}, \ldots, m_{k}$ - соответствующие кратности, $m_{1}+\cdots+m_{k}=m$, тогда

$$
\mathfrak{P}(z)=\left(z-z_{1}\right)^{m_{1}} \ldots\left(z-z_{k}\right)^{m_{k}} .
$$

Уравнение (1) является эллиптическим уравнением порядка $2 m$, у которого главной частью оператора $\mathfrak{P}(\Delta)$ является $m$-гармонический оператор $\Delta^{m}$.

Решения уравнения (1), о которых будет идти речь в работе, будем считать принадлежащими при любом $a \in(0,1)$ пространству $W_{2}^{2 m}\left(\Pi_{a}\right)$, где $\Pi_{a}=\left\{x \in \mathbb{R}^{n}, a<\right.$ $y<1\}$. Решение $u(x, y)$ называется $L_{2}$-ограниченным, если

$$
\sup _{0<y<1}\|u(\cdot, y)\|_{L_{2}\left(\mathbb{R}^{n}\right)}<\infty
$$

решение $u(x, y)$ называется имеющим $L_{2}$-предел на границе $\Gamma_{0}=\left\{x \in \mathbb{R}^{n}, y=0\right\}$ полосы П, если существует функция $u_{0}(x) \in L_{2}\left(\mathbb{R}^{n}\right)$ такая, что

$$
\left\|u(\cdot, y)-u_{0}(\cdot)\right\|_{L_{2}\left(\mathbb{R}^{n}\right)} \rightarrow 0 \quad \text { при } \quad y \rightarrow+0 .
$$

Целью настоящей работы является доказательство следующего утверждения.

* Математический институт им. В. А. Стеклова РАН, Москва, Россия. E-mail: vpmih@mi.ras.ru 
Теорема. Для того чтобы решение и $(x, y)$ уравнения (1) имело $L_{2}$-предел на границе $\Gamma_{0}$, достаточно, чтобъ выполнялось условие

$$
\int_{\Pi}|\nabla u|^{2} y d x d y<\infty
$$

$\left(\right.$ здесъ $\left.\nabla u=\left(\nabla_{x} u, u_{y}\right)=\left(u_{x_{1}}, \ldots, u_{x_{n}}, u_{y}\right)\right)$.

В работе [1] показано (см. лемму 3 этой работы), что любое из интересующих нас решений $u(x, y)$ представимо в виде

$$
u(x, y)=U(x, y)+\sum_{i=1}^{m} u_{i}(x, y), \quad(x, y) \in \Pi,
$$

где $U(x, y)$ и $u_{i}(x, y), i=1, \ldots, m$, также являются решениями в полосе П уравнения (1), причем функция $U(x, y) \in W_{2}^{2 m}(\Pi)$ и тем самым имеет интересующий нас предел на $\Gamma_{0}$; функции $u_{i}(x, y), i=1, \ldots, m$, при любом $a$ принадлежат пространству $W_{2}^{2 m}\left(\Pi_{a}\right)$, и их преобразования Фурье по переменным $x \in \mathbb{R}^{n}$ при произвольно фиксированном $y \in(0,1)$,

$$
\tilde{u}_{i}(\xi, y)=\frac{1}{(2 \pi)^{n / 2}} \int_{\mathbb{R}^{n}} e^{-i(x, \xi)} u_{i}(x, y) d x, \quad \xi \in \mathbb{R}^{n},
$$

имеют вид (см. лемму 2 работы [1])

$$
\tilde{u}_{i}(\xi, y)=A_{i}(\xi)\left(1-\chi_{R}(\xi)\right) w_{i}(\xi, y), \quad \xi \in \mathbb{R}^{n}, \quad y \in(0,1)
$$

в котором функция $A_{i}(\xi) \in L_{2, \text { loc }}\left(\mathbb{R}^{n}\right), \chi_{R}(\xi)$ - характеристическая функция шара $\{|\xi|<R\}$, где $R>\sqrt{\max _{1 \leqslant i \leqslant m}\left|z_{i}\right|+1}$.

Система функций $\left\{w_{s}(x, y), s=1, \ldots, m\right\}$ при каждом $\xi \in \mathbb{R}^{n},|\xi|>R$, как система функций переменной $y \in(0,1)$ представляет собой специальную фундаментальную систему решений обыкновенного дифференциального уравнения

$$
\prod_{j=1}^{k}\left(\frac{d}{d y}+\lambda_{j}(\xi)\right)^{m_{j}} w(\xi, y)=0, \quad \xi \in \mathbb{R}^{n}, \quad y \in[0,1]
$$

в котором

$$
\lambda_{j}(\xi)=\lambda_{j}=\sqrt{|\xi|^{2}+z_{j}}, \quad j=1, \ldots, k, \quad|\xi|>R,
$$

причем под $\sqrt{z+z_{j}}$ понимается та ветвь функции $\sqrt{z+z_{j}}$ на комплексной $z$-плоскости, разрезанной вдоль луча $z=-z_{j}+t,-\infty<t \leqslant 0$, для которой $\left(\sqrt{z+z_{j}}-\right.$ $\sqrt{z})\left.\right|_{\operatorname{Im} z=0} \rightarrow 0$ при $\operatorname{Re} z \rightarrow+\infty$, где $\left.\sqrt{z}\right|_{\operatorname{Im} z=0, \operatorname{Re} z>0}$ есть положительное значение $\sqrt{\operatorname{Re} z}$.

Функции $w_{s}(x, y) \in C^{(0, m)}(|\xi|>R, 0 \leqslant y \leqslant 1), s=1, \ldots, m$, удовлетворяют уравнению (5) и следующим начальным условиям: при $s=1$

$$
\left.w_{1}(x, y)\right|_{y=0}=1
$$


а при $s>1$

$$
\left.w_{s}(\xi, y)\right|_{y=0}=\cdots=\left.\frac{\partial^{s-2} w_{s}(\xi, y)}{\partial y^{s-2}}\right|_{y=0}=0,\left.\quad \frac{\partial^{s-1} w_{s}(\xi, y)}{\partial y^{s-1}}\right|_{y=0}=1 .
$$

Для любого целого $s$, принадлежащего сегменту $[1, m]$, определим число $\kappa=\kappa(s)$, $1 \leqslant \kappa \leqslant k$, как номер того полуинтервала из $\left(0, m_{1}\right],\left(m_{1}, m_{1}+m_{2}\right], \ldots,\left(m_{1}+\cdots\right.$ $\left.\cdots+m_{k-1}, m\right]$ (напомним, что $m=m_{1}+\cdots+m_{k}$ ), в котором содержится $s$. Тогда (см. работу [1])

$$
\begin{aligned}
w_{s}(\xi, y)= & \frac{1}{2 \pi i} \int_{|z|=|\xi|+R} \frac{e^{z y} d z}{\left(z+\lambda_{1}\right)^{m_{1}} \ldots\left(z+\lambda_{\kappa-1}\right)^{m_{\kappa-1}}\left(z+\lambda_{\kappa}\right)^{s-\left(m_{1}+\cdots+m_{\kappa-1}\right)}}, \\
& 1 \leqslant s \leqslant m, \quad \kappa=\kappa(s) \in[1, k], \quad|\xi|>R, \quad y \in[0,1]
\end{aligned}
$$

(при любом $p=1, \ldots, k$ имеет место неравенство $\left|\lambda_{p}\right| \leqslant \sqrt{|\xi|^{2}+\left|z_{p}\right|} \leqslant|\xi|+\sqrt{\left|z_{p}\right|}<$ $|\xi|+R$, поэтому все полюсы функции, стоящей под знаком интеграла в (6), находятся в круге $\{|z|<|\xi|+R\})$.

Поскольку, как уже отмечалось выше, главным оператором в дифференциальном уравнении (1) является полигармонический оператор $\Delta^{m}$, естественно, что при больших значениях $|\xi|$ функция $w_{s}(\xi, y)$ будет достаточно близка к соответствующей функции фундаментальной системы, построенной для оператора $\Delta^{m}$, т.е. к функции $e^{-y|\xi|} y^{s-1} /(s-1)$ !, а именно справедливо следующее утверждение.

Лемма 1. Для каждого $s=1,2, \ldots, m$ существуют ограниченные на множестве $\{|\xi|>R, 0 \leqslant y \leqslant 1\}$ функиии $a_{s}(\xi, y)$ и $b_{s}(\xi, y)$ такие, что

$$
w_{s}(\xi, y)=\frac{e^{-y|\xi|} y^{s-1}}{(s-1) !}\left(1+\frac{a_{s}(\xi, y)}{|\xi|}\right), \quad|\xi| \geqslant R, \quad 0 \leqslant y \leqslant 1
$$

u

$$
\frac{\partial}{\partial y} w_{s}(\xi, y)=\frac{\partial}{\partial y}\left(\frac{e^{-y|\xi|} y^{s-1}}{(s-1) !}\right)\left(1+\frac{a_{s}(\xi, y)}{|\xi|}\right)+e^{-y|\xi|} y^{s-1} \frac{b_{s}(\xi, y)}{|\xi|} .
$$

Тем самым имеют место следуюшие равномерные по $y \in[0,1]$ асимптотические разложения при $|\xi| \rightarrow \infty:$

$$
w_{s}(\xi, y)=\frac{e^{-y|\xi|} y^{s-1}}{(s-1) !}\left(1+O\left(\frac{1}{|\xi|}\right)\right)
$$

$u$

$$
\frac{\partial}{\partial y} w_{s}(\xi, y)=\frac{\partial}{\partial y}\left(\frac{e^{-y|\xi|} y^{s-1}}{(s-1) !}\right)\left(1+O\left(\frac{1}{|\xi|}\right)\right)+e^{-y|\xi|} y^{s-1} O\left(\frac{1}{|\xi|}\right) .
$$

ДокАзАтельство. Равенство (7) установлено в работе [1] (доказательству его посвящена лемма 1 работы [1]). Докажем равенство (8). Прежде всего заметим, что при любом $j=1, \ldots, k$ для всех $|\xi| \geqslant R$

$$
\begin{aligned}
\lambda_{j}=\lambda_{j}(\xi) & =\sqrt{|\xi|^{2}+z_{j}}=|\xi| \sqrt{1+\frac{z_{j}}{|\xi|^{2}}}= \\
& =|\xi|\left(1+\frac{z_{j}}{2|\xi|^{2}}+\sum_{s=1}^{\infty} \frac{(-1)^{s}(2 s-1) ! !}{2^{s+1}(s+1) !} \frac{z_{j}^{s+1}}{|\xi|^{2(s+1)}}\right),
\end{aligned}
$$


и, следовательно, для всех $i, j=1, \ldots, k$ при $|\xi| \rightarrow \infty$ имеют место соотношения

$$
\begin{gathered}
\lambda_{i}=|\xi|+\frac{z_{i}}{2|\xi|}+O\left(\frac{1}{|\xi|^{3}}\right), \\
\lambda_{i}-\lambda_{j}=\frac{z_{i}-z_{j}}{2|\xi|}+O\left(\frac{1}{|\xi|^{3}}\right) .
\end{gathered}
$$

При доказательстве равенства (8) рассмотрим сначала случай $s \leqslant m_{1}$. В этом случае

$$
\begin{aligned}
w_{s}(\xi, y) & =\frac{1}{2 \pi i} \int_{|z|=|\xi|+R} \frac{e^{y z} d z}{\left(z+\lambda_{1}\right)^{s}}=\operatorname{res}_{z=-\lambda_{1}} \frac{e^{y z}}{\left(z+\lambda_{1}\right)^{s}}= \\
& =\frac{e^{-\lambda_{1} y} y^{s-1}}{(s-1) !}=\frac{e^{-y|\xi|} y^{s-1}}{(s-1) !} e^{\left(|\xi|-\lambda_{1}\right) y}
\end{aligned}
$$

и, следовательно,

$$
\frac{\partial}{\partial y} w_{s}(\xi, y)=\frac{\partial}{\partial y}\left(\frac{e^{-y|\xi|} y^{s-1}}{(s-1) !}\right) e^{\left(|\xi|-\lambda_{1}\right) y}+\frac{e^{-y|\xi|} y^{s-1}}{(s-1) !}\left(|\xi|-\lambda_{1}\right) e^{\left(|\xi|-\lambda_{1}\right) y}
$$

т.е. для $s \in\left[1, m_{1}\right]$ в силу соотношений (9) имеет место равенство $(8)$, в котором

$$
a_{s}(\xi, y)=\left(e^{\left(|\xi|-\lambda_{1}\right) y}-1\right)|\xi|, \quad b_{s}(\xi, y)=\frac{|\xi|\left(|\xi|-\lambda_{1}\right) e^{\left(|\xi|-\lambda_{1}\right) y}}{(s-1) !}
$$

Пусть теперь $s>m_{1}$. Делая под знаком интеграла в (6) замену переменной $z=\zeta|\xi|$, а затем обозначая $\zeta$ через $z-1$, получим

$$
\begin{aligned}
w_{s}(\xi, y) & =\frac{1}{2 \pi i|\xi|^{s-1}} \int_{|\zeta|=1+\frac{R}{|\xi|}} \frac{e^{y|\xi| \zeta} d \zeta}{\left(\zeta+\frac{\lambda_{1}}{|\xi|}\right)^{m_{1}} \ldots\left(\zeta+\frac{\lambda_{\kappa}}{|\xi|}\right)^{s-\left(m_{1}+\cdots+m_{\kappa-1}\right)}}= \\
& =\frac{e^{-y|\xi|}}{2 \pi i|\xi|^{s-1}} \int_{|z-1|=1+\frac{R}{|\xi|}} \frac{e^{y|\xi| z} d z}{\left(z-\mu_{1}\right)^{m_{1}} \ldots\left(z-\mu_{\kappa}\right)^{s-\left(m_{1}+\cdots+m_{\kappa-1}\right)}}= \\
& =\frac{e^{-y|\xi|}}{2 \pi i|\xi|^{s-1}} \int_{|z|=1} \frac{e^{y|\xi| z} d z}{\left(z-\mu_{1}\right)^{m_{1}} \ldots\left(z-\mu_{\kappa}\right)^{s-\left(m_{1}+\cdots+m_{\kappa-1}\right)}},
\end{aligned}
$$

где $\mu_{i}=1-\lambda_{i} /|\xi|, i=1, \ldots, k$, причем мы считаем число $R$ настолько большим, что согласно (9) при всех $i=1, \ldots, k$ имеет место неравенство $\left|\mu_{i}\right|<1$.

Поскольку для всех комплексных $z$ при любом целом $r>0$ справедливо равенство

$$
e^{z}=1+\frac{z}{1 !}+\cdots+\frac{z^{r}}{r !}+\frac{1}{r !} \int_{0}^{z}(z-\theta)^{r} e^{\theta} d \theta
$$

в котором интегрирование ведется, например, по лучу, соединяющему точки 0 и $z$, и, в силу того, что

$$
\frac{1}{2 \pi i} \int_{|z|=1} \frac{z^{p} d z}{\left(z-\mu_{1}\right)^{m_{1}} \ldots\left(z-\mu_{\kappa}\right)^{s-\left(m_{1}+\cdots+m_{\kappa-1}\right)}}=\left\{\begin{array}{lll}
0 & \text { при } & 1 \leqslant p \leqslant s-2 \\
1 & \text { при } & p=s-1
\end{array}\right.
$$


мы имеем

$$
\begin{aligned}
w_{s}(\xi, y)= & \frac{e^{-y|\xi|}}{2 \pi i|\xi|^{s-1}} \times \\
& \times \int_{|z|=1} \frac{\left(1+\frac{y|\xi| z}{1 !}+\cdots+\frac{(y|\xi| z)^{s-1}}{(s-1) !}+\frac{1}{(s-1) !} \int_{0}^{y|\xi| z}(y|\xi| z-\theta)^{s-1} e^{\theta} d \theta\right) d z}{\left(z-\mu_{1}\right)^{m_{1}} \ldots\left(z-\mu_{\kappa}\right)^{s-\left(m_{1}+\cdots+m_{\kappa-1}\right)}}= \\
= & \frac{e^{-y|\xi|} y^{s-1}}{(s-1) !}\left(1+\frac{y|\xi|}{2 \pi i} \int_{|z|=1} \frac{z^{s} \int_{0}^{1}(1-t)^{s-1} e^{y|\xi| z t} d t d z}{\left(z-\mu_{1}\right)^{m_{1}} \ldots\left(z-\mu_{\kappa}\right)^{s-\left(m_{1}+\cdots+m_{\kappa-1}\right)}}\right) .
\end{aligned}
$$

Следовательно,

$$
\begin{aligned}
\frac{\partial w_{s}(\xi, y)}{\partial y}=\frac{\partial}{\partial y} & \left(\frac{e^{-y|\xi|} y^{s-1}}{(s-1) !}\right)\left(1+\frac{y|\xi|}{2 \pi i} \int_{|z|=1} \frac{z^{s} \int_{0}^{1}(1-t)^{s-1} e^{y|\xi| z t} d t d z}{\left(z-\mu_{1}\right)^{m_{1}} \ldots\left(z-\mu_{\kappa}\right)^{s-\left(m_{1}+\cdots+m_{\kappa-1}\right)}}\right)+ \\
& +\frac{e^{-y|\xi|} y^{s-1}}{(s-1) !} \frac{|\xi|}{2 \pi i} \int_{|z|=1} \frac{z^{s} \int_{0}^{1}(1-t)^{s-1} e^{y|\xi| z t} d t d z}{\left(z-\mu_{1}\right)^{m_{1}} \ldots\left(z-\mu_{\kappa}\right)^{s-\left(m_{1}+\cdots+m_{\kappa-1}\right)}}+ \\
& +\frac{e^{-y|\xi|} y^{s-1}}{(s-1) !} \frac{y|\xi|^{2}}{2 \pi i} \int_{|z|=1} \frac{z^{s+1} \int_{0}^{1}(1-t)^{s-1} t e^{y|\xi| z t} d t d z}{\left(z-\mu_{1}\right)^{m_{1}} \ldots\left(z-\mu_{\kappa}\right)^{s-\left(m_{1}+\cdots+m_{\kappa-1}\right)}} .
\end{aligned}
$$

Таким образом, для того чтобы установить справедливость формулы (8), достаточно показать, что равномерно по $y \in[0,1]$ и по $t \in[0,1]$ при $|\xi| \rightarrow \infty$ имеют место соотношения

$$
\begin{aligned}
& \int_{|z|=1} \frac{z^{s} e^{y|\xi| z t} d z}{\left(z-\mu_{1}\right)^{m_{1}} \ldots\left(z-\mu_{\kappa}\right)^{s-\left(m_{1}+\cdots+m_{\kappa-1}\right)}}=O\left(\frac{1}{|\xi|^{2}}\right) \\
& \int_{|z|=1} \frac{z^{s+1} e^{y|\xi| z t} d z}{\left(z-\mu_{1}\right)^{m_{1}} \ldots\left(z-\mu_{\kappa}\right)^{s-\left(m_{1}+\cdots+m_{\kappa-1}\right)}}=O\left(\frac{1}{|\xi|^{4}}\right) .
\end{aligned}
$$

Введем временные обозначения $m_{1}=n_{1}, \ldots, s-\left(m_{1}+\cdots+m_{\kappa-1}\right)=n_{\kappa}$ и вычислим при целом $b=s$ или $b=s+1$ интеграл

$$
\frac{1}{2 \pi i} \int_{|z|=1} \frac{z^{b} e^{y|\xi| z t} d z}{\left(z-\mu_{1}\right)^{n_{1}} \ldots\left(z-\mu_{\kappa}\right)^{n_{\kappa}}}=\sum_{j=1}^{\kappa} \operatorname{res}_{z=\mu_{j}} \frac{z^{b} e^{y|\xi| z t}}{\left(z-\mu_{1}\right)^{n_{1}} \ldots\left(z-\mu_{\kappa}\right)^{n_{\kappa}}} .
$$

Имеем

$$
\begin{aligned}
\operatorname{res}_{z=\mu_{j}} & \frac{z^{b} e^{y|\xi| z t}}{\left(z-\mu_{1}\right)^{n_{1}} \ldots\left(z-\mu_{\kappa}\right)^{n_{\kappa}}}=\frac{1}{\left(n_{j}-1\right) !} \frac{d^{n_{j}-1}}{d z^{n_{j}-1}} \times \\
& \times\left.\left(\frac{z^{b} e^{y|\xi| z t}}{\left(z-\mu_{1}\right)^{n_{1}} \ldots\left(z-\mu_{j-1}\right)^{n_{j-1}}\left(z-\mu_{j+1}\right)^{n_{j+1}} \ldots\left(z-\mu_{\kappa}\right)^{n_{\kappa}}}\right)\right|_{z=\mu_{j}}= \\
= & \sum_{p+q} C_{p q r} \frac{d^{p} e^{y|\xi| z t}}{d z^{p}} \frac{d^{q} z^{b}}{d z^{q}} \times 1 \\
& \quad \times\left.\frac{d^{r}}{d z^{r}}\left(\frac{1}{\left(z-\mu_{1}\right)^{n_{1}} \ldots\left(z-\mu_{j-1}\right)^{n_{j-1}}\left(z-\mu_{j+1}\right)^{n_{j+1}} \ldots\left(z-\mu_{\kappa}\right)^{n_{\kappa}}}\right)\right|_{z=\mu_{j}},
\end{aligned}
$$

где $C_{p q r}-$ соответствующие мультиномиальные коэффициенты. 
Из равенств (9) следует, что при $|\xi| \rightarrow \infty$

$$
\begin{aligned}
& \mu_{j}=\mu_{j}(\xi)=1-\frac{\lambda_{j}(\xi)}{|\xi|}=O\left(\frac{1}{|\xi|^{2}}\right), \\
& \mu_{j}-\mu_{i}=\frac{z_{j}-z_{i}}{2|\xi|^{2}}+O\left(\frac{1}{|\xi|^{4}}\right), \quad i, j=1, \ldots, k
\end{aligned}
$$

поэтому при $|\xi| \rightarrow \infty$

$$
\begin{gathered}
\left.\frac{d^{p} e^{y|\xi| z t}}{d z^{p}}\right|_{z=\mu_{j}}=y^{p}|\xi|^{p} t^{p} e^{y|\xi| t \mu_{j}}=O\left(|\xi|^{p}\right), \\
\frac{d^{q} z^{b}}{d z^{q}}=O\left(\frac{1}{|\xi|^{2(b-q)}}\right),
\end{gathered}
$$

Далее,

$$
\left.\frac{d^{r}}{d z^{r}}\left(\frac{1}{\left(z-\mu_{1}\right)^{n_{1}} \ldots\left(z-\mu_{j-1}\right)^{n_{j-1}}\left(z-\mu_{j+1}\right)^{n_{j+1}} \ldots\left(z-\mu_{\kappa}\right)^{n_{\kappa}}}\right)\right|_{z=\mu_{j}}
$$

есть линейная комбинация с не зависящими от $\xi$ коэффициентами выражений вида

$$
\frac{1}{\left(\mu_{j}-\mu_{1}\right)^{c_{1}} \ldots\left(\mu_{j}-\mu_{j-1}\right)^{c_{j-1}}\left(\mu_{j}-\mu_{j+1}\right)^{c_{j+1}} \ldots\left(\mu_{j}-\mu_{\kappa}\right)^{c_{\kappa}}},
$$

в которых $c_{1}+\cdots+c_{j-1}+c_{j+1}+\cdots+c_{\kappa}=s-n_{j}+r$, поэтому

$$
\begin{gathered}
\left.\frac{d^{r}}{d z^{r}}\left(\frac{1}{\left(z-\mu_{1}\right)^{n_{1}} \ldots\left(z-\mu_{j-1}\right)^{n_{j-1}}\left(z-\mu_{j+1}\right)^{n_{j+1}} \ldots\left(z-\mu_{\kappa}\right)^{n_{\kappa}}}\right)\right|_{z=\mu_{j}}= \\
=O\left(|\xi|^{2\left(s-n_{j}+r\right)}\right), \quad|\xi| \rightarrow \infty .
\end{gathered}
$$

Следовательно,

$$
\begin{gathered}
\operatorname{res}_{z=\mu_{j}} \frac{z^{b} e^{y|\xi| z t}}{\left(z-\mu_{1}\right)^{n_{1}} \ldots\left(z-\mu_{\kappa}\right)^{n_{\kappa}}}=O\left(|\xi|^{p-2(b-q)+2\left(s-n_{j}+r\right)}\right)= \\
=O\left(|\xi|^{2(p+q+r)-p-2 b+2 s-2 n_{j}}\right)=O\left(|\xi|^{2 n_{j}-2-p-2 b+2 s-2 n_{j}}\right)= \\
=O\left(|\xi|^{-2-p+2(s-b)}\right)=O\left(|\xi|^{-2+2(s-b)}\right), \quad|\xi| \rightarrow \infty .
\end{gathered}
$$

Поскольку полученное равенство для вычета в точке $\mu_{j}$ от $j$ не зависит, для вычетов в других полюсах $\mu_{1}, \ldots, \mu_{j-1}, \mu_{j+1}, \ldots, \mu_{\kappa}$ имеют место такие же равенства. Поэтому в силу (12) равномерно по $y \in[0,1]$ и по $t \in[0,1]$

$$
\int_{|z|=1} \frac{z^{b} e^{y|\xi| z t} d z}{\left(z-\mu_{1}\right)^{n_{1}} \ldots\left(z-\mu_{\kappa}\right)^{n_{\kappa}}}=O\left(|\xi|^{-2+2(s-b)}\right), \quad|\xi| \rightarrow \infty,
$$

откуда при $b=s$ и $b=s+1$ вытекают соотношения (10) и (11). Лемма доказана. 
Обозначим через $\mathfrak{A}=\left\|a_{i j}\right\|_{i, j=1, \ldots, m}$ симметрическую вещественную матрицу размера $(m \times m)$ с элементами

$$
a_{i j}=a_{j i}=\int_{0}^{\infty} t \frac{\left(e^{-t} t^{i-1}\right)_{t}}{(i-1) !} \frac{\left(e^{-t} t^{j-1}\right)_{t}}{(j-1) !} d t, \quad i, j=1, \ldots, m .
$$

Нетрудно проверить, что

$$
\begin{aligned}
a_{11} & =\frac{1}{4}, \\
a_{i j} & =\frac{\Gamma(i+j-2)\left(i+j-2-(i-j)^{2}\right)}{2^{i+j} \Gamma(i) \Gamma(j)}, \quad i+j>2 .
\end{aligned}
$$

Квадратичная форма этой матрицы, т.е. функция $\mathfrak{K}=\mathfrak{K}(B), B=\left(B_{1}, \ldots, B_{m}\right) \in$ $\mathbb{C}^{m}$, имеет вид

$$
\mathfrak{K}(B)=(\mathfrak{A} B, B)_{\mathbb{C}^{m}}=\sum_{i=1}^{m} \sum_{j=1}^{m} a_{i j} B_{i} \bar{B}_{j}=\sum_{i=1}^{m} \sum_{j=1}^{m} B_{i} \bar{B}_{j} \int_{0}^{\infty} t \frac{\left(e^{-t} t^{i-1}\right)_{t}}{(i-1) !} \frac{\left(e^{-t} t^{j-1}\right)_{t}}{(j-1) !} d t .
$$

Функция $\mathfrak{K}$ является вещественнозначной, неотрицательной,

$$
\begin{aligned}
\mathfrak{K}(B) & =\int_{0}^{\infty} \sum_{i=1}^{m} B_{i} \sqrt{t} \frac{\left(e^{-t} t^{i-1}\right)_{t}}{(i-1) !} \sum_{j=1}^{m} \bar{B}_{j} \sqrt{t} \frac{\left(e^{-t} t^{j-1}\right)_{t}}{(j-1) !} d t= \\
& =\left\|\sum_{i=1}^{m} B_{i} \sqrt{t} \frac{\left(e^{-t} t^{i-1}\right)_{t}}{(i-1) !}\right\|_{L_{2}(0, \infty)}^{2} .
\end{aligned}
$$

Справедливо следующее утверждение.

ЛЕмма 2. Минимальное собственное значение а матрицы $\mathfrak{A}$ положительно, m.e.

$$
\inf _{\|B\|_{\mathbb{C}^{m}=1}} \mathfrak{K}(B)=\mathfrak{a}>0 .
$$

ДоКАЗАТЕЛЬСТво. Предположим, что в противоположность утверждению леммы $\mathfrak{a}=0$. Тогда существует последовательность векторов $B^{k}=\left(B_{1}^{k}, \ldots, B_{m}^{k}\right)$, $\left\|B^{k}\right\|_{\mathbb{C}^{m}}=1, k=1,2, \ldots$, для которой $\mathfrak{K}\left(B^{k}\right) \rightarrow 0$ при $k \rightarrow \infty$. Эту последовательность можно за счет возможного ее прорежения считать сходящейся в $\mathbb{C}^{m} \mathrm{~K}$ некоторому вектору $B^{0}=\left(B_{1}^{0}, \ldots, B_{m}^{0}\right),\left\|B^{0}\right\|_{\mathbb{C}^{m}}=1$. Тогда равномерно по $t \in[0, \infty)$

$$
\sum_{i=1}^{m} B_{i}^{k} \sqrt{t} \frac{\left(e^{-t} t^{i-1}\right)_{t}}{(i-1) !} \underset{k \rightarrow \infty}{\longrightarrow} \sum_{i=1}^{m} B_{i}^{0} \sqrt{t} \frac{\left(e^{-t} t^{i-1}\right)_{t}}{(i-1) !}
$$

и, следовательно, в силу (14)

$$
\sum_{i=1}^{m} B_{i}^{0} \sqrt{t} \frac{\left(e^{-t} t^{i-1}\right)_{t}}{(i-1) !} \equiv 0, \quad t \in[0, \infty)
$$

тем самым

$$
\sum_{i=1}^{m} B_{i}^{0} \frac{e^{-t} t^{i-1}}{(i-1) !}=C, \quad t \in[0, \infty)
$$


при некоторой постоянной $C$, т.е. имеет место равенство

$$
\sum_{i=1}^{m} B_{i}^{0} \frac{t^{i-1}}{(i-1) !}=C e^{t}, \quad t \in[0, \infty),
$$

которое возможно только при $C=B_{1}^{0}=\cdots=B_{m}^{0}=0$. Но это противоречит условию $\left\|B^{0}\right\|_{\mathbb{C}^{m}}=1$.

ЛЕмма 3. Если решение и $(x, y)$ уравнения (1) удовлетворяет условию

$$
\int_{\Pi}\left|u_{y}\right|^{2} y d x d y<\infty
$$

то функции $A_{i}(\xi)$ из равенств (4) таковы, что

$$
\frac{A_{i}(\xi)}{\left(1+|\xi|^{2}\right)^{(i-1) / 2}} \in L_{2}\left(\mathbb{R}^{n}\right), \quad i=1, \ldots, m .
$$

ДоказАтЕЛЬСтво. Функция $U(x, y)$ из представления решения $u(x, y)$ по формуле (3) принадлежит $W_{2}^{2 m}(\Pi)$, отсюда $\int_{\Pi}\left|U_{y}\right|^{2} y d x d y=C_{1}<\infty$, где $C_{1}$ - некоторая зависящая от $u(x, y)$ постоянная, следовательно, в силу (3)

$$
\int_{\Pi}\left|\sum_{i=1}^{m} u_{i y}(x, y)\right|^{2} y d x d y=C_{2}=C_{2}(u)<\infty .
$$

Из (4) вытекает, что для любого $y \in(0,1)$

$$
\begin{aligned}
\int_{\mathbb{R}^{n}}\left|\sum_{i=1}^{m} u_{i y}(x, y)\right|^{2} d x & =\int_{|\xi|>R}\left|\sum_{i=1}^{m} A_{i}(\xi) w_{i y}(\xi, y)\right|^{2} d \xi= \\
& =\int_{|\xi|>R}\left|\sum_{i=1}^{m} B_{i}(\xi)\left(|\xi|^{i-1} w_{i y}(\xi, y)\right)\right|^{2} d \xi= \\
& =\sum_{i=1}^{m} \sum_{j=1}^{m} \int_{|\xi|>R} B_{i}(\xi) \overline{B_{j}(\xi)}|\xi|^{i-1}|\xi|^{j-1} w_{i y}(\xi, y) \overline{w_{j y}(\xi, y)} d \xi
\end{aligned}
$$

где

$$
B_{i}(\xi)=\frac{A_{i}(\xi)}{|\xi|^{i-1}}, \quad|\xi|>R, \quad i=1, \ldots, m .
$$

Следовательно, согласно (18)

$$
\begin{aligned}
C_{2}= & \int_{\Pi}\left|\sum_{i=1}^{m} u_{i y}(x, y)\right|^{2} y d x d y= \\
= & \int_{|\xi|>R} \sum_{i=1}^{m} \sum_{j=1}^{m} B_{i}(\xi) \overline{B_{j}(\xi)} \int_{0}^{1} y|\xi|^{i-1}|\xi|^{j-1} w_{i y}(\xi, y) \overline{w_{j y}(\xi, y)} d y d \xi= \\
= & \int_{|\xi|>R} \sum_{i=1}^{m} \sum_{j=1}^{m} B_{i}(\xi) \overline{B_{j}(\xi)} \frac{|\xi|^{i-1}|\xi|^{j-1}}{|\xi|^{2}} \times \\
& \quad \times\left.\left.\int_{0}^{|\xi|} \eta\left(\frac{\partial w_{i}(\xi, y)}{\partial y}\right)\right|_{y=\eta /|\xi|} \overline{\left(\frac{\partial w_{j}(\xi, y)}{\partial y}\right)}\right|_{y=\eta /|\xi|} d \eta d \xi .
\end{aligned}
$$


Из формул (7) и (8) леммы 1 вытекает, что

$$
\begin{aligned}
&\left.w_{i}(\xi, y)\right|_{y=\eta /|\xi|}= \frac{e^{-\eta} \eta^{i-1}}{|\xi|^{i-1}(i-1) !}\left(1+\frac{a_{i}(\xi, \eta /|\xi|)}{|\xi|}\right) \\
& \frac{\left.\partial w_{i}(\xi, y)\right|_{y=\eta /|\xi|}=}{\partial y}\left(\left.\left.\frac{\partial}{\partial y}\left(\frac{e^{-y|\xi|} y^{i-1}}{(i-1) !}\right)\right|_{y=\eta /|\xi|}\left(1+\frac{a_{i}(\xi, y)}{|\xi|}\right)\right|_{y=\eta /|\xi|}+\right. \\
&+\left.\left.\left(e^{-y|\xi|} y^{i-1}\right)\right|_{y=\eta /|\xi|} \frac{b_{i}(\xi, y)}{|\xi|}\right|_{y=\eta /|\xi|}= \\
&=\frac{|\xi|}{|\xi|^{i-1}} \frac{\partial}{\partial \eta}\left(\frac{e^{-\eta} \eta^{i-1}}{(i-1) !}\right)\left(1+\frac{a_{i}(\xi, \eta /|\xi|)}{|\xi|}\right)+ \\
&+\left(e^{-\eta} \eta^{i-1}\right) \frac{b_{i}(\xi, \eta /|\xi|)}{|\xi|^{i}}
\end{aligned}
$$

(здесь $\left.\eta \in \mathbb{R}^{1},|\xi|>R, i=1, \ldots, m\right)$. Следовательно, как продолжение равенства (20) имеем

$$
\begin{aligned}
C_{2}= & \int_{|\xi|>R} \sum_{i=1}^{m} \sum_{j=1}^{m} B_{i}(\xi) \overline{B_{j}(\xi)} \times \\
& \times \int_{0}^{|\xi|} \eta\left[\frac{\partial}{\partial \eta}\left(\frac{e^{-\eta} \eta^{i-1}}{(i-1) !}\right)\left(1+\frac{a_{i}(\xi, \eta /|\xi|)}{|\xi|}\right)+\left(e^{-\eta} \eta^{i-1}\right) \frac{b_{i}(\xi, \eta /|\xi|)}{|\xi|}\right] \times \\
& \times\left[\frac{\partial}{\partial \eta}\left(\frac{e^{-\eta} \eta^{j-1}}{(j-1) !}\right)\left(1+\frac{\overline{a_{j}(\xi, \eta /|\xi|)}}{|\xi|}\right)+\left(e^{-\eta} \eta^{j-1}\right) \frac{\overline{b_{j}(\xi, \eta /|\xi|)}}{|\xi|}\right] d \eta d \xi
\end{aligned}
$$

При любом $\xi,|\xi|>R$, комплексная $(m \times m)$-матрица $\mathfrak{A}(\xi)=\left\|a_{i j}(\xi)\right\|_{i, j=1, \ldots, m}$, в которой

$$
\begin{aligned}
a_{i j}(\xi)= & \int_{0}^{|\xi|} \eta\left[\frac{\partial}{\partial \eta}\left(\frac{e^{-\eta} \eta^{i-1}}{(i-1) !}\right)\left(1+\frac{a_{i}(\xi, \eta /|\xi|)}{|\xi|}\right)+\left(e^{-\eta} \eta^{i-1}\right) \frac{b_{i}(\xi, \eta /|\xi|)}{|\xi|}\right] \times \\
& \times\left[\frac{\partial}{\partial \eta}\left(\frac{e^{-\eta} \eta^{j-1}}{(j-1) !}\right)\left(1+\frac{\overline{a_{j}(\xi, \eta /|\xi|)}}{|\xi|}\right)+\left(e^{-\eta} \eta^{j-1}\right) \frac{\overline{b_{j}(\xi, \eta /|\xi|)}}{|\xi|}\right] d \eta,
\end{aligned}
$$

очевидно, является самосопряженной, $\mathfrak{A}(\xi)=\mathfrak{A}^{*}(\xi)$. Далее, при любых $i=1, \ldots, m$ функции $e^{-\eta} \eta^{i-1}$ и $\left|\left(e^{-\eta} \eta^{i-1}\right)_{\eta}\right|$ интегрируемы на $[0, \infty)$, а функции $a_{i}(\xi, \eta /|\xi|)$ и $b_{i}(\xi, \eta /|\xi|)$ ограничены на множестве $\{|\xi|>R, 0 \leqslant \eta<|\xi|\}$, что вытекает из утверждения леммы 1 об ограниченности функций $a_{i}(\xi, y)$ и $b_{i}(\xi, y)$ на множестве $\{|\xi|>R, 0 \leqslant y \leqslant 1\}$. Таким образом,

$$
\begin{aligned}
a_{i j}(\xi)= & \int_{0}^{\infty} \eta \frac{\left(e^{-\eta} \eta^{i-1}\right)_{\eta}}{(i-1) !} \frac{\left(e^{-\eta} \eta^{j-1}\right)_{\eta}}{(j-1) !} d \eta- \\
& -\int_{|\xi|}^{\infty} \eta \frac{\left(e^{-\eta} \eta^{i-1}\right)_{\eta}}{(i-1) !} \frac{\left(e^{-\eta} \eta^{j-1}\right)_{\eta}}{(j-1) !} d \eta+O\left(\frac{1}{|\xi|}\right), \quad|\xi| \rightarrow \infty,
\end{aligned}
$$


т.е. согласно (13) при всех $i, j=1, \ldots, m$

$$
a_{i j}(\xi) \rightarrow a_{i j}, \quad|\xi| \rightarrow \infty
$$

где, напомним, $a_{i j}$ - элементы матрицы $\mathfrak{A}$.

Следовательно, минимальное собственное значение $\mathfrak{a}(\xi)$ матрицы $\mathfrak{A}(\xi)$ при $|\xi| \rightarrow \infty$ стремится к минимальному собственному значению $\mathfrak{a}$ матрицы $\mathfrak{A}$, которое согласно лемме 2 положительно. Таким образом, существует такое $R_{1} \geqslant R$, что для всех $\xi$, $|\xi| \geqslant R_{1}$, имеет место неравенство $\mathfrak{a}(\xi) \geqslant \mathfrak{a} / 2$, и тем самым квадратичная форма $\mathfrak{K}(\xi, B)=(\mathfrak{A}(\xi) B, B)_{\mathbb{C}^{m}}, B \in \mathbb{C}^{m}$, матрицы $\mathfrak{A}(\xi)$ удовлетворяет неравенству

$$
\inf _{\|B\|_{\mathbb{C} m}=1} \mathfrak{K}(\xi, B) \geqslant \frac{\mathfrak{a}}{2} \quad \text { при }|\xi| \geqslant R_{1},
$$

из которого следует неравенство

$$
\mathfrak{K}(\xi, B) \geqslant \frac{\mathfrak{a}}{2}\|B\|_{\mathbb{C}^{m}}^{2} \quad \text { для всех } \quad \xi,|\xi| \geqslant R_{1}, \quad \text { и всех } \quad B \in \mathbb{C}^{m} .
$$

Продолжим равенство (21). С помощью (22) получим неравенство

$$
\begin{aligned}
C_{2} & \geqslant \int_{|\xi| \geqslant R_{1}} \sum_{i=1}^{m} \sum_{j=1}^{m} a_{i j}(\xi) B_{i}(\xi) \overline{B_{j}(\xi)} d \xi=\int_{|\xi| \geqslant R_{1}} \mathfrak{K}(\xi, B(\xi)) d \xi \geqslant \\
& \geqslant \frac{\mathfrak{a}}{2} \int_{|\xi| \geqslant R_{1}} \sum_{i=1}^{m}\left|B_{i}(\xi)\right|^{2} d \xi,
\end{aligned}
$$

из которого в силу (19) вытекает, что для всех $i=1, \ldots, m$

$$
\int_{|\xi| \geqslant R_{1}} \frac{\left|A_{i}(\xi)\right|^{2}}{|\xi|^{i-1}} d \xi \leqslant \frac{2 C_{2}}{\mathfrak{a}}
$$

и тем более

$$
\int_{|\xi| \geqslant R_{1}} \frac{\left|A_{i}(\xi)\right|^{2}}{\left(1+|\xi|^{2}\right)^{(i-1) / 2}} d \xi \leqslant \frac{2 C_{2}}{\mathfrak{a}} .
$$

Утверждение леммы 3 теперь следует из известного включения $A_{i}(\xi) \in L_{2, \text { loc }}\left(\mathbb{R}^{n}\right)$, $i=1, \ldots, m$.

Лемма 4. Пусть и(x,y) - решение уравнения (1). Тогда условия (2) и (16) эквивалентны.

ДоказАтельство. То, что из условия (2) вытекает условие (16), очевидно, поскольку $|\nabla u|^{2}=\left|\nabla_{x} u\right|^{2}+\left|u_{y}\right|^{2} \geqslant\left|u_{y}\right|^{2}$ для всех $(x, y) \in \Pi$.

Пусть выполнено условие (16). Для доказательства соотношения (2) следует проверить, что

$$
\int_{\Pi}\left|\nabla_{x} u\right|^{2} y d x d y<\infty .
$$


Поскольку функция $U(x, y)$ в $(3)$ принадлежит $W_{2}^{1}(\Pi)$, для этого достаточно показать, что при любом $i=1, \ldots, m$

$$
\int_{\Pi}\left|\nabla_{x} u_{i}\right|^{2} y d x d y<\infty
$$

другими словами, с учетом (4) установить, что для всех $i=1, \ldots, m$

$$
\int_{0}^{1} y d y \int_{|\xi|>R}\left|A_{i}(\xi)\right|^{2}|\xi|^{2}\left|w_{i}(\xi, y)\right|^{2} d \xi<\infty .
$$

Для доказательства неравенства (24) воспользуемся равенством (7) (или (7а)) из леммы 1 ; при этом, как и при доказательстве леммы 3 , достаточно проверить, что при некотором (достаточно большом) $R_{1} \geqslant R$ для всех $i=1, \ldots, m$

$$
\int_{0}^{1} y d y \int_{|\xi|>R_{1}}\left|A_{i}(\xi)\right|^{2}|\xi|^{2} e^{-2 y|\xi|} y^{2(i-1)} d \xi<\infty .
$$

Проверка последнего неравенства осуществляется непосредственно с помощью леммы 3:

$$
\begin{array}{rl}
\int_{0}^{1} y & d y \int_{|\xi|>R_{1}}\left|A_{i}(\xi)\right|^{2}|\xi|^{2} e^{-2 y|\xi|} y^{2(i-1)} d \xi= \\
= & \int_{|\xi|>R_{1}}\left|A_{i}(\xi)\right|^{2}|\xi|^{2} \int_{0}^{1} e^{-2 y|\xi|} y^{2 i-1} d y d \xi= \\
= & \int_{|\xi|>R_{1}}\left|A_{i}(\xi)\right|^{2}|\xi|^{2} \frac{1}{(2|\xi|)^{2 i}} \int_{0}^{2|\xi|} e^{-t} t^{2 i-1} d t d \xi \leqslant \\
\leqslant \frac{1}{2^{2 i}} \int_{|\xi|>R_{1}} \frac{\left|A_{i}(\xi)\right|^{2}}{|\xi|^{2(i-1)}} \int_{0}^{\infty} e^{-t} t^{2 i-1} d t d \xi \leqslant \\
\leqslant \frac{\Gamma(2 i)}{4} \int_{\mathbb{R}^{n}} \frac{\left|A_{i}(\xi)\right|^{2}}{\left(1+|\xi|^{2}\right)^{i-1}} d \xi<\infty, \quad i=1, \ldots, m .
\end{array}
$$

Для доказательства теоремы нам понадобится еще одно вспомогательное утверждение.

ЛЕмма 5. Если функиия $A_{i}(\xi)$ из равенства (4) при $i=2, \ldots, m$ удовлетворяет условию

$$
\lim _{N \rightarrow \infty} \int_{N<|\xi|<2 N} \frac{\left|A_{i}(\xi)\right|^{2}}{\left(1+|\xi|^{2}\right)^{i-1}} d \xi=0,
$$

то функция $u_{i}(x, y)$ из (3) при этих $i$ имеет на границе $\Gamma_{0} L_{2}$-предел, равный нулю.

Утверждение этой леммы является одним из утверждений леммы 7 работы [1].

ДокАЗАТЕЛЬСтво теОРЕмы. Пусть для решения $u(x, y)$ уравнения $(1)$ выполнено условие (2). Тогда по лемме 4 выполнено условие (16). Следовательно, согласно лемме 3 функции $A_{i}(\xi)$ в равенствах (4) таковы, что

$$
\frac{A_{i}(\xi)}{\left(1+|\xi|^{2}\right)^{(i-1) / 2}} \in L_{2}\left(\mathbb{R}^{n}\right), \quad i=1, \ldots, m .
$$


Отсюда при $i=1$ имеем $A_{1}(\xi) \in L_{2}\left(\mathbb{R}^{n}\right)$. Покажем, что функция $u_{1}(x, y)$ из формулы (3) имеет $L_{2}$-предел на $\Gamma_{0}$, равный функции

$$
f_{1}(x)=\frac{1}{(2 \pi)^{n / 2}} \int_{\mathbb{R}^{n}} e^{i(x, \xi)} A_{1}(\xi)\left(1-\chi_{R}(\xi)\right) d \xi .
$$

Действительно,

$$
\begin{aligned}
\left\|u_{1}(\cdot, y)-f_{1}(\cdot)\right\|_{L_{2}\left(\mathbb{R}^{n}\right)}^{2} & =\left\|\tilde{u}_{1}(\cdot, y)-A_{1}(\cdot)\left(1-\chi_{R}(\cdot)\right)\right\|_{L_{2}\left(\mathbb{R}^{n}\right)}^{2}= \\
& =\int_{\mathbb{R}^{n}}\left|A_{1}(\xi)\left(1-\chi_{R}(\xi)\right)\left(1-e^{-y|\xi|}\right)\right|^{2} d \xi \rightarrow 0, \quad y \rightarrow+0,
\end{aligned}
$$

поскольку подынтегральная функция для почти всех $\xi \in \mathbb{R}^{n}$ стремится к нулю при $y \rightarrow+0$ и имеет интегрируемую мажоранту $\left|A_{1}(\xi)\left(1-\chi_{R}(\xi)\right)\right|^{2}$.

Из (25) вытекает, что

$$
\frac{\left|A_{i}(\xi)\right|^{2}}{\left(1+|\xi|^{2}\right)^{(i-1) / 2}} \in L_{1}\left(\mathbb{R}^{n}\right), \quad i=2, \ldots, m .
$$

Следовательно, для $i=2, \ldots, m$

$$
\lim _{N \rightarrow \infty} \int_{N<|\xi|<2 N} \frac{\left|A_{i}(\xi)\right|^{2}}{\left(1+|\xi|^{2}\right)^{i-1}} d \xi=0,
$$

а тогда по лемме 5 функции $u_{i}(x, y), i=2, \ldots, m$, имеют нулевые $L_{2}$-пределы на $\Gamma_{0}$. Функция $U(x, y) \in W_{2}^{1}(\Pi)$, поэтому $u(x, y)$ имеет $L_{2}$-предел на $\Gamma_{0}$. Теорема доказана.

В заключение скажем несколько слов об условии (2). Впервые оно появилось в работах Литтлвуда и Пэли [2], [3]. В этих работах было доказано, что аналитическая в единичном круге $\{|z|<1\}$ функция $u(z)$ одного комплексного переменного $z=x+i y$ тогда и только тогда имеет $L_{2}$-предел на границе, когда выполнено условие

$$
\int_{|z|<1}\left|u^{\prime}\right|^{2}(1-|z|) d x d y<\infty .
$$

Этот результат был перенесен в работе [4] на случай, когда функция $u(x)$ является решением в $n$-мерной ограниченной области $Q$ линейного (не обязательно однородного) эллиптического уравнения второго порядка; при этом коэффициенты уравнения и граница $\partial Q$ области $Q$ предполагались достаточно гладкими. В работе [4] доказано, что необходимым и достаточным условием существования $L_{2}$-предела на границе функции $u(x)$ является условие

$$
\int_{Q}|\nabla u(x)|^{2} r(x) d x<\infty
$$

где $r(x)$ - расстояние от точки $x \in Q$ до границы $\partial Q$.

Однако условие типа условия Литтвуда-Пэли не может быть критерием существования $L_{2}$-предела на границе области у всех решений линейного эллиптического 
уравнения, если порядок этого уравнения выше 2. Доказанная в настоящей работе теорема утверждает, что для всех решений уравнения (1) условие (2) является достаточным условием для существования $L_{2}$-предела на $\Gamma_{0}$. Также можно показать, что для любого уравнения (1) при $m>1$ существуют решения, имеющие $L_{2}$-предел на $\Gamma_{0}$, для которых условие $(2)$ не выполняется. При построении соответствующего примера ограничимся случаем $\mathfrak{P}(z)=z^{2}$, т.е. случаем бигармонического уравнения.

Легко проверить, что функция

$$
v(x, y)=\frac{1}{(2 \pi)^{n / 2}} \int_{\mathbb{R}^{n}} e^{i(x, \xi)} \frac{e^{-y|\xi|} d \xi}{\left(1+|\xi|^{2}\right)^{(n-2) / 4}\left(1+\ln \left(1+|\xi|^{2}\right)\right)^{1 / 2}}, \quad(x, y) \in \Pi,
$$

является гармонической в П, следовательно, функция

$$
u(x, y)=y v(x, y), \quad(x, y) \in \Pi,
$$

является в П бигармонической.

Для любого $y \in(0,1]$

$$
\begin{aligned}
\|u(\cdot, y)\|_{L_{2}\left(\mathbb{R}^{n}\right)}^{2} & =y^{2} \int_{\mathbb{R}^{n}} \frac{e^{-2 y|\xi|} d \xi}{\left(1+|\xi|^{2}\right)^{(n-2) / 2}\left(1+\ln \left(1+|\xi|^{2}\right)\right)}= \\
& =y^{2} \omega_{n} \int_{0}^{\infty} \frac{\rho^{n-1} e^{-2 y \rho} d \rho}{\left(1+\rho^{2}\right)^{(n-2) / 2}\left(1+\ln \left(1+\rho^{2}\right)\right)}= \\
& =\omega_{n} \int_{0}^{\infty} \frac{r^{n-1} e^{-2 r} d r}{\left(y^{2}+r^{2}\right)^{(n-2) / 2}\left(1+\ln \left(1+r^{2} / y^{2}\right)\right)},
\end{aligned}
$$

где $\omega_{n}=2 \pi^{n / 2} / \Gamma(n / 2)$. Подынтегральное выражение в последнем интеграле стремится к нулю при $y \rightarrow+0$ для всех $r>0$ и мажорируется интегрируемой функцией $r e^{-2 r}$, отсюда $\|u(\cdot, y)\|_{L_{2}\left(\mathbb{R}^{n}\right)}^{2} \rightarrow 0$ при $y \rightarrow+0$, таким образом, функция $u(x, y)$ имеет $L_{2}$-предел на $\Gamma_{0}$, равный нулю.

Проверим, что условие (2) для функции $u(x, y)$ не выполняется. Для всех $y \in(0,1]$

$$
\begin{aligned}
\int_{\mathbb{R}^{n}}|\nabla u(x, y)|^{2} d x & =2 y^{2} \int_{\mathbb{R}^{n}} \frac{|\xi|^{2} e^{-2 y|\xi|} d \xi}{\left(1+|\xi|^{2}\right)^{(n-2) / 2}\left(1+\ln \left(1+|\xi|^{2}\right)\right)}= \\
& =2 y^{2} \omega_{n} \int_{0}^{\infty} \frac{r^{n+1} e^{-2 y r} d r}{\left(1+r^{2}\right)^{(n-2) / 2}\left(1+\ln \left(1+r^{2}\right)\right)}
\end{aligned}
$$

поэтому

$$
\begin{aligned}
& \int_{\Pi} y|\nabla u(x, y)|^{2} d x d y=2 \omega_{n} \int_{0}^{1} y^{3} \int_{0}^{\infty} \frac{r^{n+1} e^{-2 y r} d r}{\left(1+r^{2}\right)^{(n-2) / 2}\left(1+\ln \left(1+r^{2}\right)\right)} d y= \\
&=2 \omega_{n}\left\{\frac{3}{8} \int_{1}^{\infty} \frac{r^{n-3} d r}{\left(1+r^{2}\right)^{(n-2) / 2}\left(1+\ln \left(1+r^{2}\right)\right)}+\right. \\
&+\int_{0}^{1} y^{3} \int_{0}^{1} \frac{r^{n+1} e^{-2 y r} d r}{\left(1+r^{2}\right)^{(n-2) / 2}\left(1+\ln \left(1+r^{2}\right)\right)} d y- \\
&\left.-\int_{1}^{\infty} \frac{e^{-2 r}\left(1 / 2 r+3 / 4 r^{2}+3 / 4 r^{3}+3 / 8 r^{4}\right)}{\left(1+r^{2}\right)^{(n-2) / 2}\left(1+\ln \left(1+r^{2}\right)\right)} d r\right\}=\infty
\end{aligned}
$$


поскольку первый интеграл в фигурной скобке равен $\infty$, а последующие два интеграла сходятся.

Отметим, что аналогичный пример полигармонической функции в круге (двумерный случай) приведен в работе [5]. Другое достаточное условие существования $L_{2}$-предельного значения на границе области у решений весьма общих эллиптических уравнений имеется в работе [6].

Благодарности. Работа выполнена при финансовой поддержке РФФИ (грант № 07-01-00144а) и Программы поддержки ведущих научных школ (грант НШ$3224.2008 .1)$.

\section{Список литературы}

[1] В. П. Михайлов, Матем. сб., 190:10 (1999), 17-48.

[2] J. E. Littlewood, R. Paley, Proc. London Math. Soc., 42:1 (1936), 52-89.

[3] J. E. Littlewood, R. Paley, Proc. Lond. Math. Soc., 43 (1937), 105-126.

[4] В. П. Михайлов, Матем. сб., 101(143):2(10) (1976), 163-188.

[5] В.П. Михайлов, “Об одном достаточном условии существования предельных значений полигармонических функций на границе области", Тр. межд. семинара “Дифференциальные уравнения и их приложения" (Самара, 1998).

[6] Я. А. Ройтберг, Докл. АН СССР, 238:6 (1978), 1303-1306.

Поступила в редакцию 13.03.2008

5 Теоретическая и математическая физика, т. 157, № 3, 2008 г. 